\title{
Determining the exponent of the unloading curve when flattening a sphere
}

\author{
Petr Ogar*, Elena Ugryumova, and Denis Gorokhov \\ Bratsk State University, Makarenko 40, 665709 Bratsk, Russia
}

\begin{abstract}
To study the flattening of the sphere, it is proposed to use the kinetic indentation diagram by the plane. Given the known values of the reduced elastic modulus, applied force, maximum and residual deformation, it is possible to determine the contact area. It is indicated that in this regard, the exponent of the unloading curve of a pre-loaded sphere with a flat rigid surface plays an important role. The analysis of methods for determining the unloading curves of unloading for the finite element models, taking into account strain hardening, is carried out. It is shown that dependences of the unloading curves during flattening on the relative indentation in the form and the range of values differ from the similar ones during indentations of the sphere. The dependence between the exponents of the unloading curves for the force and for the area is determined. The range of correct use of the results of the finite element analysis of a hemisphere for rough surfaces is indicated. The exponent of the unloading curve after flattening the spherical segment from the half-space property is determined.
\end{abstract}

\section{Introduction}

Determination of contact characteristics at the joint of rough surfaces is a difficult problem, both from the point of view of describing rough surfaces, and from the point of view of the mechanics of contact of individual asperities. Therefore, when solving such problems, various methods of mathematical modeling are usually used, which leads to various models of rough surfaces: statistical, discrete, fractal, fractal-discrete, etc. [1-7]. All of these models assume a contact between an equivalent rough and smooth surface. As follows from [4], a rough surface can be represented as a set of bodies of regular geometric shape (rods, cones, pyramids, spheres, ellipsoids), for which the solution of contact problems is known. Moreover, their height distribution in the rough layer should corresponds to the surface bearing curve. A spherical model of a single asperity is considered optimal.

When a sphere comes into contact with a flat surface, depending on the ratio of mechanical properties, it is possible an indentation or flattening of a sphere. In most cases, the contact is elastoplastic. The results of studies of elastoplastic contacts of a sphere and a plane are given in $[8,9]$, which indicate the absence of a closed solution. An exception is the engineering methods for sphere indentation presented in $[10,11]$ based on the concept

\footnotetext{
*Corresponding author: ogar@brstu.ru
} 
of "plastic hardness" and the linear dependence of the residual deformation on the applied load during the sphere indentation and having experimental confirmation.

The complexity of the problem led to the creation of the finite element models (FEM) of the contact of a sphere and a plane. To simplify the finite element solutions of elastoplasticity problems, mathematical models are used to describe the true stress-strain curves. The most commonly used elastoplastic Hollomon body [12-15, etc.], for which

$$
S=\left\{\begin{array}{l}
E \varepsilon, \quad \varepsilon \leq \varepsilon_{y}, \quad \sigma \leq \sigma_{y} \\
E \varepsilon_{y}^{1-n} \varepsilon^{n}, \quad \varepsilon>\varepsilon_{y}, \quad \sigma>\sigma_{y}
\end{array}\right.
$$

where $\varepsilon_{y}=S_{y} / E ; S_{y} \approx \sigma_{y}, S_{y}$ and $\sigma_{y}$ are the true and conditional yield strength; $\varepsilon$ is the deformation; $E$ is the elastic modulus, $n$ is the hardening exponent.

In [16], an engineering method is given for calculating the contact characteristics during flattening of a sphere by modernizing the Drozd-Matlin model [10, 17]. In this case, the results of modeling were used to determine the residual deformation during flattening of the sphere. To determine the contact area, the kinetic indentation method proposed by S.I. Bulychev [18] to determine the elastic modulus of the material. In Fig. 1, it is shown a typical diagram of kinetic indentation, which is usually represented in coordinates $P-h$, or $P-\bar{h}$, where $P$ is the load, $\bar{h}=h / R$ is the relative indentation of the indenter.

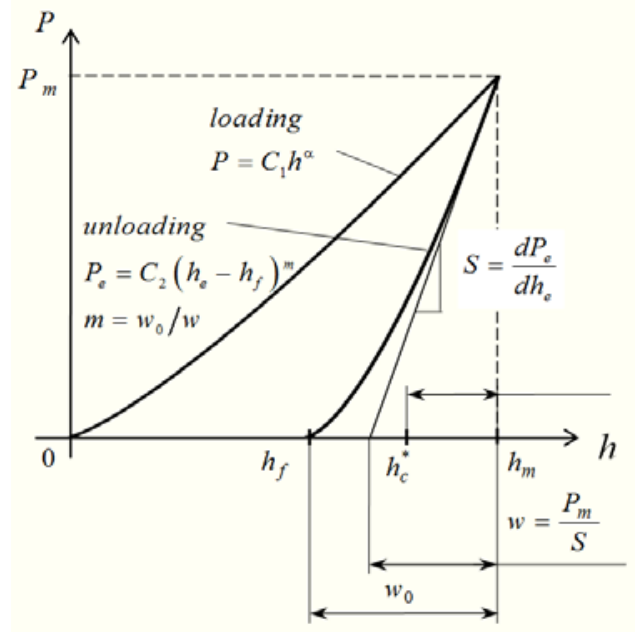

Fig. 1. The kinetic indentation diagram.

The loading curve can be described by the equations

$$
P=P_{m} h_{m}^{-\alpha} h^{\alpha}=P_{m} \bar{h}_{m}^{-\alpha} \bar{h}^{\alpha}=C_{l} \bar{h}^{\alpha},
$$

and the unloading curve is

$$
P_{e}=P_{m}\left(\frac{h_{e}-h_{f}}{h_{m}-h_{f}}\right)^{m}=P_{m}\left(\frac{\bar{h}_{e}-\bar{h}_{f}}{\bar{h}_{m}-\bar{h}_{f}}\right)^{m},
$$

where $C_{l}=P_{m} \bar{h}_{m}^{-\alpha}, \bar{h}_{m}=h_{m} / R, R$ is the sphere radius; $\alpha=1 \ldots 1.5, m=1.2 \ldots 1.5$ is the exponents.

The method is based on the equation for the contact stiffness of the initial section of the unloading curve [18]

$$
S=\frac{d P_{e}}{d h_{e}}=\frac{2}{\sqrt{\pi}} E^{*} \sqrt{A}
$$


where $A$ is the projection of the contact area, $E^{*}$ is the reduced modulus of elasticity.

Taking into account expression (3), we have

$$
\frac{P_{m} m}{h_{m}-h_{f}}=\frac{2}{\sqrt{\pi}} E^{*} \sqrt{A} .
$$

Given the known values of the reduced modulus of elasticity, applied force, maximum and permanent deformation, it is possible to determine the contact area. In this regard, an important role is played by the exponent of the unloading curve of a pre-loaded sphere by a flat rigid surface, the refinement of the values of which is the purpose of this work.

\section{Analysis of methods for determining the exponent of the unloading curve}

As follows from Fig. 1, with kinetic indentation, the exponent of the unloading curve is determined by the ratio

$$
m=\frac{w_{0}}{w}
$$

where $w=P_{m} / S$.

According to [19], as per concept of "effective indenter shape", the value of the exponent of the unloading curve is expected in the range $1.2 \ldots 1.5$, which is confirmed by the experimental values are given. In [11], the parameter $m$ was determined by calculation using the results of the finite element analysis:

$$
m=\frac{3-2 c^{2} \bar{h}}{2-c^{2} \bar{h}},
$$

where $c^{2}=h_{c} / h=c^{2}\left(\varepsilon_{y}, n, \bar{h}\right)$, determined according to [12]; $h_{c}$ is the contact depth.

Fig. 2 and Fig. 3 show the dependence of the exponent of the unloading curve on the relative indentation of the rigid sphere for different values of $\varepsilon_{y}$ and $n$. As follows from Fig. 2a with an increase in the elastic properties of the material (values of $n$ and $\varepsilon_{y}$ ), the value of the exponent of the curve increases. For the relative indentation $\bar{h}=0 \ldots 0.2$ characteristic of the tribomechanics of rough surfaces, the values of the exponent of the unloading curve are in the range of $1.426 \ldots$ 1.5.

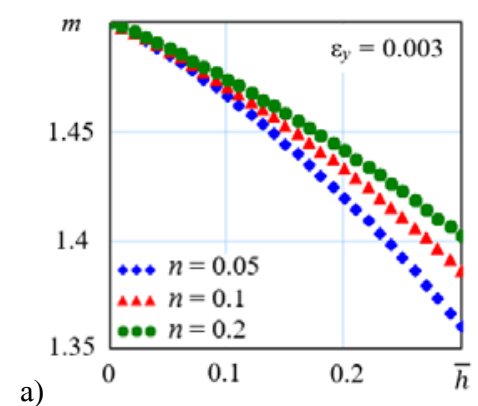

b)

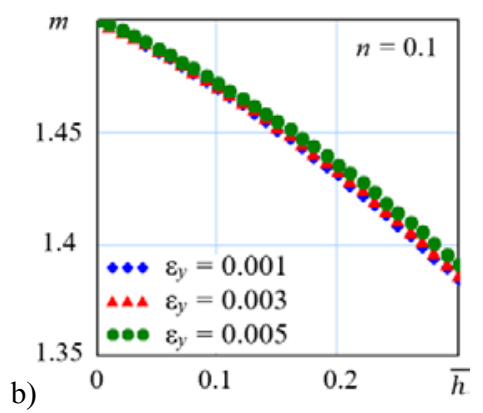

Fig. 2. Dependencies $m-\bar{h}$ at fixed values $\varepsilon_{y}$ (a) and at fixed values of $n(b)$.

Fig. $2 b$ shows the dependences of the exponent of the unloading curve on the relative indentation of the rigid sphere at fixed values of $n$ for different values $\varepsilon_{y}$. In this case, the range of scatter of $m$ values is much narrower than in the previous case. 


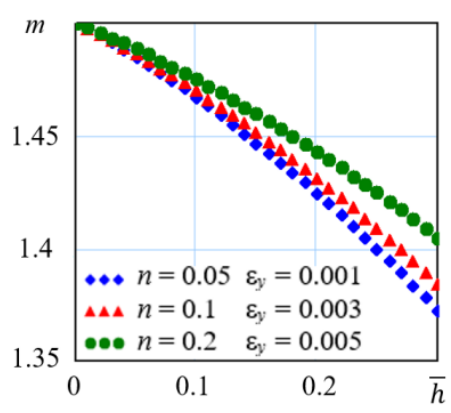

Fig. 3. Dependencies $m-\bar{h}$ for materials with increasing elastic properties.

For the FEM of sphere flattening, taking into account strain hardening $[14,15]$, the unloading curve is described by an equation similar to equation (3) for the unloading curve when the sphere is indented:

$$
P_{e}=P_{m}\left(\frac{w_{e}-w_{\text {res }}}{w_{m}-w_{\text {res }}}\right)^{m_{p}}=P_{m}\left(\frac{\bar{w}_{e}-\bar{w}_{\text {res }}}{\bar{w}_{m}-\bar{w}_{\text {res }}}\right)^{m_{p}},
$$

where $\bar{w}_{m}=w_{m} / R, w_{\text {res }}$ is the residual displacement when unloading the sphere (analogue $h_{f}$ ) defined by the equation:

$$
\frac{w_{r e s}}{w_{m}}=\left(1-\left(\omega_{m}^{*}\right)^{-d}\right)^{2}
$$

unloading curve exponent:

$$
m_{p}=1.5 \cdot\left(\omega_{m}^{*}\right)^{e}
$$

where for FEM [14]:

$$
\begin{aligned}
& e_{p}=e_{p}(n)=0.07071 n^{2}-0.0411 n-0.02955, \\
& d=d(n)=-0.22471 n^{2}-0.17406 n+0.39877
\end{aligned}
$$

for FEM [15]:

$$
\begin{gathered}
e_{p}=e_{p}(n, v)=0.0519 n^{2}+0.0705 n v-0.0401 n-0.0718 v-0.00596, \\
d=d(n, v)=-0.114 n^{2}-0.237 n+0.219 v+0.331
\end{gathered}
$$

here $n$ is the hardening exponent, $v$ is the Poisson's ratio, $w_{m}^{*}=w_{m} / w_{c}, w_{c}$ is the critical displacement at which plastic deformation begins.

Fig. 4 shows the dependence of the exponent of the unloading curve on the relative displacement after flattening the sphere with a rigid surface.

For relative flattening $\bar{w}=0 . .0 .2$, the values of the exponent of the unloading curve $m_{p}$ are in the range $1.045 \ldots 1.5$, which significantly differ from the analogous range when the sphere is introduced. A sharp drop in values $m_{p}$ occurs in the range $0 \leq \bar{w} \leq 0.005$. 
a)

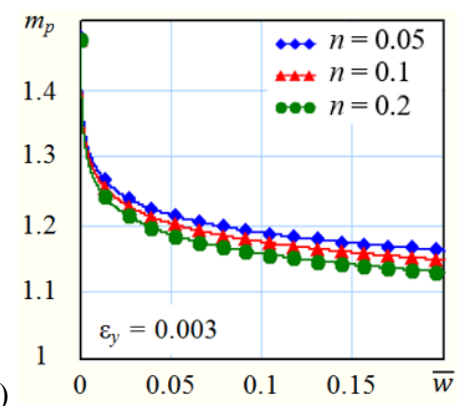

b)

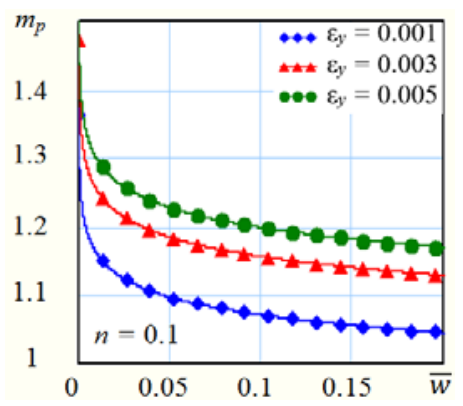

Fig. 4. Dependencies $m_{p}-\bar{w}$ at fixed values $\varepsilon_{y}$ (a) and at fixed values of $n$ (b) for FEM [14].

When spherical asperities are flattened, the dependencies $m_{p}-\bar{w}$ are concave, while when spherical asperities are indented, they are convex. In addition, Fig. 4a shows that the incorrect results are obtained for more plastic materials - the values $m_{p}$ are larger than for more elastic ones.

Similar results for FEM [15] are shown in Fig. 5, at which the values of the exponent of the unloading curve are in the range $1.11 \ldots 1.5$.
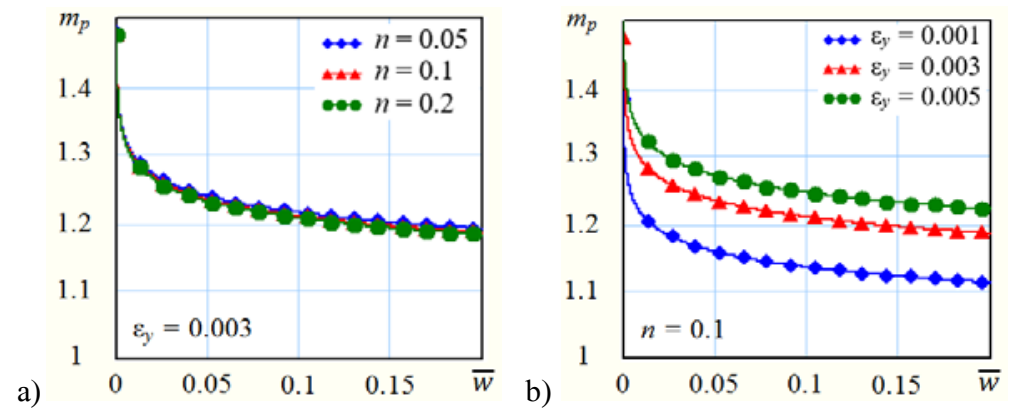

Fig. 5. Dependencies $m_{p}-\bar{w}$ at fixed values $\varepsilon_{y}$ (a) and at fixed values of $n$ (b) for FEM [15].

Similar results on the determination of the contact areas upon indentation and flattening of spherical asperities obtained in [20] suggest similar values of the exponentials of the unloading curves during indentation and flattening, which is based on the analysis of equation (3).

The above analysis presupposes additional studies of FEM [14, 15].

\section{Research methodology}

Investigations were carried out in the following directions: the "updated" parameters of the flattening FEM were compared with the calculated ones - the forces in contact and the exponent of the unloading curves; the relationship between the exponents of unloading for force and area was determined; the exponent of the unloading curve was determined when a spherical segment flattens from the property of a half-space.

Let us give expressions for the force and area of the FEM contact [14]:

$$
\frac{P}{P_{c}}=B_{i}\left(\frac{w_{m}}{w_{c}}\right)^{\gamma_{i}}=B_{i}\left(w_{m}^{*}\right)^{\gamma_{i}},
$$




$$
\frac{A}{A_{c}}=C_{i}\left(\frac{w_{m}}{w_{c}}\right)^{\lambda_{i}}=C_{i}\left(w_{m}^{*}\right)^{\lambda_{i}},
$$

where $B_{i}=B_{i}(n), \gamma_{i}=\gamma_{i}(n), C_{i}=C_{i}(n), \lambda_{i}=\lambda_{i}(n)$ are the parameters that depend on the hardening exponent $n$ for different ranges $w^{*} ; w_{c}, P_{c}$ and $A_{c}$ are the critical values of the parameters corresponding to the onset of plastic deformation are determined from the relations of the Hertz theory [6]:

$$
\bar{w}_{c}=\frac{w_{c}}{R}=\frac{\left(\pi K_{y} \varepsilon_{y}\right)^{2}}{4}, A_{c}=\pi R^{2} \bar{w}_{c}
$$

where $K_{y}=(0.756-0.450 \cdot v)^{-1} ; \varepsilon_{y}=\sigma_{y} / E^{*}, E^{*}$ is the reduced modulus of elasticity;

$$
\begin{aligned}
& P_{c}=4 E^{*} R^{1 / 2} w_{c}^{3 / 2} / 3, \\
& \bar{P}_{c}=\frac{P_{c}}{E^{*} R^{2}}=\frac{\left(\pi K_{y} \varepsilon_{y}\right)^{3}}{6}
\end{aligned}
$$

From equation (5) taking into account equations (9) - (12) and (16), it follows that

$$
\bar{P}_{a}=\bar{P}_{c} C_{2}^{0.5}\left(w^{*}\right)^{1+0.5 \lambda_{2}-e_{p}}\left[2\left(w^{*}\right)^{-d}-\left(w^{*}\right)^{-2 d}\right]
$$

Neglecting the term of a lower order of smallness in parentheses from (20), we have

$$
\bar{P}_{a}=\bar{P}_{c} C_{2}^{0.5}\left(w^{*}\right)^{1+0.5 \lambda_{2}-e_{p}} .
$$

Fig. 6 shows the dependences $\bar{P}-\bar{w}$, calculated by equations (21) and (15), from which good agreement follows.

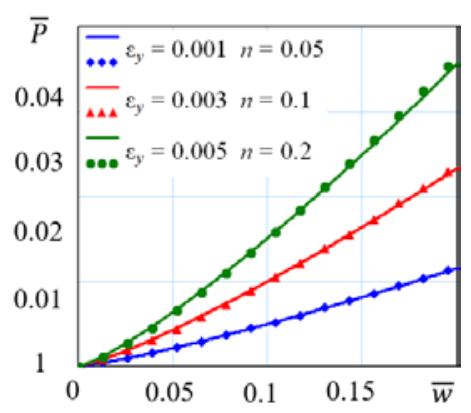

Fig. 6. Relative force versus relative displacement. Points correspond to equation (19), lines - to equation (15).

Thus, from equations (15) and (21), it follows that

$$
B_{2} \approx 2 \cdot C_{2}^{0.5}, \gamma_{2} \approx 1+0.5 \lambda_{2}-e_{p}-d \text {. }
$$

On the other hand, equation (5) makes it possible to determine the exponent of the unloading curve

$$
m_{p}=\frac{3 \cdot C_{2}^{0.5}}{2 \cdot B_{2}}\left(w^{*}\right)^{1+0.5 \lambda_{2}-\gamma_{2}}\left[2\left(w^{*}\right)^{-d}-\left(w^{*}\right)^{-2 d}\right] .
$$

Fig. 7 shows the results of calculating the exponents of the unloading curve using equation (23) obtained from equation (20). Despite the good agreement of efforts in Fig. 6, the spread of exponent values calculated by different methods increases with increasing relative displacement. In addition, there is an incorrect result - the exponent value of a more elastic material is less than for a less elastic one. 


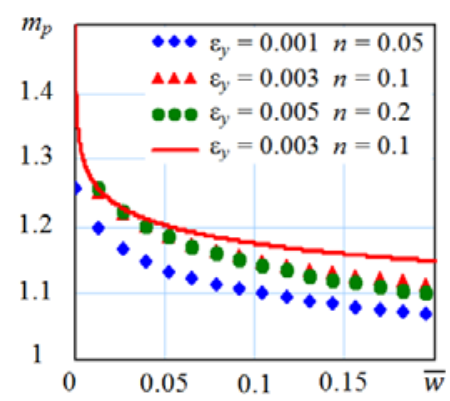

Fig. 7. Dependencies $m_{p}-\bar{w}$. Dots - equation (23), line - equation (10).

Let us carry out similar research of the exponent of the unloading curve for the FEM [15], in which the equations are used for the force and contact area:

$$
\begin{gathered}
A^{*}=\omega^{*}\left(1+\exp \left(1-\left(\omega^{*}\right)^{\alpha}\right)^{-1}\right), \\
P^{*}=\left(\omega^{*}\right)^{3 / 2}\left(1-\exp \left(1-\left(\omega^{*}\right)^{\beta}\right)^{-1}\right), \\
\alpha=\alpha(n, v)=0.179 n^{2}-0.420 n+0.184 v+0.2432, \\
\beta=\beta(n, v)=-0.159 n^{2}+0.0731 v+0.183 .
\end{gathered}
$$

Equations (24) - (27) given in [15] are defined for $0 \leq n \leq 1$ and $1<\omega^{*} \leq 120$. In the subsequent work of the authors [21] it is indicated that $1<\omega^{*}$.

From equation (5), taking into account (9), (24) and (25), for the exponent of the unloading curve, we obtain

$$
m_{p}=\frac{3}{2} \frac{\sqrt{1+\exp \left(1-\left(\omega^{*}\right)^{\alpha}\right)^{-1}}}{1-\exp \left(1-\left(\omega^{*}\right)^{\beta}\right)^{-1}}\left[2\left(\omega^{*}\right)^{d}-\left(\omega^{*}\right)^{-2 d}\right] .
$$

The exponential values of the unloading curves calculated by equation (28) do not agree with the values obtained by equation (10). The reason for this can only be the errors of the model described by equations (24) - (27).

Let us consider the relationship between the exponents of the unloading curves for force and area. Similarly to equation (8), we have

$$
A_{e}=A_{m}\left(\frac{w_{e}-w_{\text {res }}}{w_{m}-w_{\text {res }}}\right)^{m_{a}} .
$$

When reloading the unloaded sphere, the displacement value [6] is

$$
w_{0}=w_{e}-w_{\text {res }}=\frac{P K_{\beta 0}}{\pi a E^{*}},
$$

where $a=(A / \pi)^{0.5}$ is the contact area radius; when flattening the sphere $K_{\beta 0}=2$.

From equation (30), taking into account (29), we obtain

$$
P_{e}=K_{w}\left(w_{e}-w_{r e s}\right)^{1+0.5 m_{a}},
$$


where $K_{w}=\frac{\pi E^{*}}{K_{\beta 0}}\left(\frac{A_{m}}{\pi\left(w_{e}-w_{\text {res }}\right)^{m_{a}}}\right)^{0.5}$.

Differentiate equation (31) is

$$
\frac{d P_{e}}{d w_{e}}=K_{w}\left(1+0.5 m_{a}\right)\left(w_{e}-w_{r e s}\right)^{0.5 m_{a}} .
$$

When

$$
m_{p}=\frac{w_{e}-w_{\text {res }}}{P_{e}} \cdot \frac{d P_{e}}{d w_{e}}=1+0.5 m_{a} .
$$

Fig. 8 shows a comparison of dependencies calculated by equations (10) and (33).

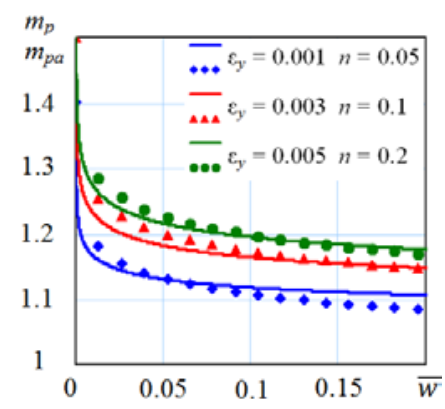

Fig. 8. Dependences of the exponents of the unloading curve on the relative displacement. Dots equation (10), line - equation (33).

The use of a hemisphere in the finite element modeling of flattening the asperities of a rough surface is not a good option, since there are no such asperities whose height is equal to the radius. The average angle of inclination for rough surface asperities is several degrees. In work [22], it is indicated that if we represent an individual asperity in the form of a spherical segment with a base diameter of $2 a$, which is located on a cylindrical base, then the base will act as a semi-infinite rigid body when its height exceeds $6 a$.

Therefore, we believe that the correct use of the results of the finite element analysis during flattening is possible for $a / R<0.17$.

Let's introduce the parameter $C^{a}$ (analogue $c^{2}=h_{c} / h$ when indentation the sphere, equation (7)):

$$
C^{a}=\frac{A(w)}{A^{\prime}(w)},
$$

where $A(w)$ is the contact area is determined by the equation (16); $A^{\prime}(w)$ is the crosssectional area of the sphere at the level $w$;

$$
A^{\prime}(w)=\pi\left(2 R w-w^{2}\right)
$$

When

$$
C^{a}=\frac{A(w)}{A^{\prime}(w)}=\frac{\pi R w_{c} C_{2}\left(w^{*}\right)^{\lambda_{2}}}{\pi R w \cdot(2-\bar{w})}=\frac{C_{2}\left(w^{*}\right)^{\lambda_{2}-1}}{2-\bar{w}}
$$

By analogy with equation (7), we obtain

$$
m_{p}=\frac{3-2 C^{a}}{2-C^{a}},
$$

This approach is possible when the spherical segment is in a half-space with the same material. 


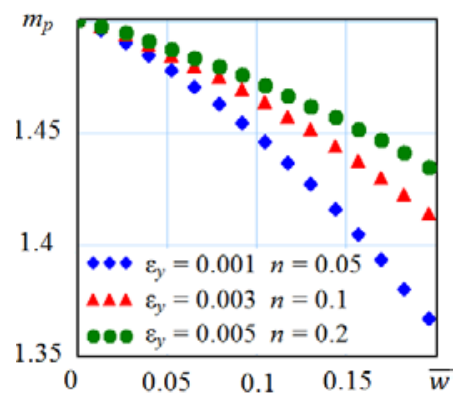

Fig. 9. Dependences of the exponents of the unloading curve on the relative displacement when flattening a spherical segment with the properties of a half-space.

Fig. 9 shows the dependences $m_{p}-\bar{w}$ calculated by equation (37), which are convex. The unloading curve exponential range is close to that for sphere indentation.

\section{Conclusion}

1. To study the flattening of a sphere, it is proposed to use a diagram of kinetic indentation by a plane. With known values of the reduced elastic modulus, applied force, maximum and residual deformation, it is possible to determine the contact area. In this regard, the exponent of the unloading curve of a pre-loaded sphere with a flat rigid surface plays an important role.

2. The analysis of methods for determining the exponents of unloading curves for the finite element models that take into account strain hardening is carried out. It is shown that the dependences of the exponents of the unloading curves during flattening on the relative indentation in the shape and range of values significantly differ from those for the indentation of the sphere.

In this case, incorrect results were obtained - for more plastic materials, the values $m_{p}$ are larger than for more elastic ones. This should be attributed to the disadvantages of the model.

3. The research was carried out in the following directions: the "updated" parameters of the FEM of flattening of the sphere were compared with the calculated ones - the forces in contact and the exponential curves of unloading; the relationship between the exponents of unloading for force and area was determined; the exponent of the unloading curve was determined for flattening a spherical segment with the properties of a half-space.

4. Good agreement was obtained for the dependences $\bar{P}-\bar{w}$ calculated by equations (20) and (15), which indicates a qualitative relationship between the parameters of the FEM [14]. In this case, the spread in the values of the exponentials of the unloading curves calculated by different methods with an increase in the relative displacement can be considered satisfactory.

Similar values of the exponents of the unloading curves for the FEM [15], calculated by expression (28), do not agree with the values obtained by equation (10), which can only be explained by model errors.

5. The relationship between the exponents of the unloading curves for the force and for the area has been established, which also confirms the qualitative relationship of the FEM parameters [14].

6. Correct use of the results of the finite element analysis of a hemisphere for rough surfaces is possible for $a / R<0.17$. 
To determine the exponent of the unloading curve when flattening a spherical segment from the half-space property, a parameter $C^{a}$ is introduced. In this case, the range of values of the exponents of the unloading curve and the shape of the dependencies are close to those for the indentation of the sphere.

\section{References}

1. J.A. Greenwood, J.B.R. Williamson, Proc. Roy.Soc. A295, 301 (1966)

2. A. Majumdar, B. Bhushan, ASME: J. of Tribology, 112, 205-216 (1990)

3. W. Yan, K. Komvopoulos, J. of Appl. Physics, 84 (7), 3617-3624 (1998)

4. N.B. Demkin, Contact of rough surfaces (1970)

5. I.V. Kragel'skij, N.M. Dobychin, V.S. Kombalov, Basics of calculation the friction and wear (1974)

6. P.M. Ogar, D.B. Gorokhov, A.S. Kozhevnikov, Contact tasks in hermetic sealing studies of fixed joints (2017)

7. P. Ogar, S. Belokobylsky, D. Gorokhov, Contact and Fracture Mechanics (2018)

8. H. Gheadnia, S. A. Pope, R. L. Jackson, D. B. Marghitu, Tribiology International, 93, Part A, 78 (2016)

9. H. Ghaednia, X. Wang, S. Saha, Y. Xu, A. Sharma, R. L. Jackson, ASME. Appl. Mech. Rev., 69(6): 060804 (2017)

10. M.S. Drozd, M.M. Matlin, Yu.I. Sidyakin, Engineering calculations of elastoplastic contact deformation (1986)

11. P.M. Ogar, V.A. Tarasov, Adv. Mat. Res., 664, 625 (2013)

12. X. Hernot, O. Bartier, Y. Bekouche, G. Mauvoisin, R. El Abdi, Int. J. Solids and Struct., 43, 4136 (2006)

13. J.-M. Collin, G. Mauvoisin, P. Pilvin, Materials and Desing, 31, 636 (2010)

14. J.H. Zhao, S. Nagao, Z.I. Zhang, Int. J. of Mech. Sciences, 56, 70-76 (2012)

15. B. Zhao, S. Zhang, Q. Wang, Q. Zhang, P. Wang, Int. J. of Mechanical Sciences, 94, 20-26 (2015)

16. P. Ogar, E. Ugryumova, D. Gorokhov, A. Zhuk, Materials Today: Proceedings, DOI: 10.1016/j.matpr.2020.08.174 (to be published)

17. M.M. Matlin, S.L. Lebskij, A.I. Mozgunova, Regularities of Elastoplastic Contact in Problems of Surface Plastic Hardening (2007)

18. S.I. Bulychev, V.P. Alekhin, Testing materials by continuous indentation of the indenter (1990)

19. W.C. Oliver, G.M. Pharr, Journal of Materials Research, 19 (1), 3-20 (2004)

20. P. Ogar, D. Gorokhov, E. Ugryumova, Int. J. of Engineering and Technology(UAE), 7 (2.23), pp. 188-191 (2018)

21. B. Zhao, S. Zhang, Z. Qiu, Tribology Int., 92, 57-66 (2015)

22. S. Saha, R.L. Jackson, Tribology - Materials, Surfaces \& Interfaces, DOI: $10.1080 / 17515831.2019 .1663396$ (2019) 